

\title{
Adaptive Dictionary Learning For Competitive Classification Of Multiple Sclerosis Lesions
}

\author{
Hrishikesh Deshpande, Pierre Maurel, Christian Barillot
}

\section{To cite this version:}

Hrishikesh Deshpande, Pierre Maurel, Christian Barillot. Adaptive Dictionary Learning For Competitive Classification Of Multiple Sclerosis Lesions. International Symposium on BIOMEDICAL IMAGING: From Nano to Macro, Apr 2015, New-York, United States. hal-01121110

\section{HAL Id: hal-01121110 https://inria.hal.science/hal-01121110}

Submitted on 27 Feb 2015

HAL is a multi-disciplinary open access archive for the deposit and dissemination of scientific research documents, whether they are published or not. The documents may come from teaching and research institutions in France or abroad, or from public or private research centers.
L'archive ouverte pluridisciplinaire HAL, est destinée au dépôt et à la diffusion de documents scientifiques de niveau recherche, publiés ou non, émanant des établissements d'enseignement et de recherche français ou étrangers, des laboratoires publics ou privés. 


\title{
ADAPTIVE DICTIONARY LEARNING FOR COMPETITIVE CLASSIFICATION OF MULTIPLE SCLEROSIS LESIONS
}

\author{
Hrishikesh Deshpande, Pierre Maurel, Christian Barillot
}

\author{
Inria, VisAGeS INSERM U746, IRISA UMR CNRS 6074, University of Rennes-1, Rennes, France
}

\begin{abstract}
Sparse representations allow modeling data using a few basis elements of an over-complete dictionary and have been used in many image processing applications. We propose to use a sparse representation and an adaptive dictionary learning paradigm to automatically classify Multiple Sclerosis (MS) lesions from MRI. In particular, we investigate the effects of learning dictionaries specific to the lesions and individual healthy brain tissues, which include White Matter (WM), Gray Matter (GM) and Cerebrospinal Fluid (CSF). The dictionary size plays a major role in data representation but it is an even more crucial element in the case of competitive classification. We present an approach that adapts the size of the dictionary for each class, depending on the complexity of the underlying data. The proposed algorithm is evaluated on clinical data demonstrating improved classification.
\end{abstract}

Index Terms - Sparse Representations, Adaptive Dictionary Learning, Magnetic Resonance Imaging

\section{INTRODUCTION}

An important variety of natural images can be represented as linear combinations of a few atoms of a learned dictionary. Over the last few years, researchers have shown that the sparse representation of the images in such a way produces promising results in image denoising and classification, including face recognition and texture classification [1-3]. Recently, its applications in disease detection have started evolving $[4,5]$. In this paper, we present a novel method using sparse representations and dictionary learning framework to classify MS lesions.

Multiple sclerosis is an autoimmune disease affecting the central nervous system and is characterized by the structural damages of axons. MRI is the best paraclinical method for the diagnosis of MS and treatment efficacy. Manual segmentation of MS lesions, however, is a laborious and time consuming task, and is prone to high intra- and inter-expert variability. Several MS lesion segmentation methods have been proposed over the last decades, with an objective of handling large variety of MR data and which can provide results that correlate well with expert analysis [6]. These methods either use lesion properties or tissue segmentation to help lesion segmentation.
We investigate the performance of automatic lesion classification by taking into account the tissue specific information. In the past, Weiss et al. [4] treated lesions as outliers and achieved MS lesion segmentation using a single dictionary learned with the help of all image patches. Deshpande $e t$ $a l$. [5] reported improvements by learning class specific dictionaries for the lesion and healthy tissue patches. However, we can further enrich this model by using dictionaries specific to the different tissue types, such as WM, GM and CSF, as opposed to learning a single dictionary for healthy tissue patches. We explore the fact that various tissues as well as lesions appear in different intensity patterns in distinct MR modality images. For example, WM appears as the brightest tissue in T1-weighted image, but the darkest in T2-weighted images. Therefore, learning class specific dictionaries for individual tissues should further discriminate between lesion and non-lesion classes. We consider multiple MR modality images and learn the tissue specific dictionaries for WM, GM, CSF and lesion classes, to better classify MS lesions.

The dictionaries learned for each class are aimed at better representation of an individual class. However, if there exists differences in the data-complexity between classes, the relative under- or over-representation of either class will lead to worse classification. One idea for better classification could be to learn the dictionaries with adaptive sizes, in order to take into account the data variability between different classes. Thus, in addition to the dictionary learning strategy mentioned above, we also investigate the effect of modifying the dictionary sizes, leading to the proposition of adaptive dictionary learning. The basic idea is to learn the class specific dictionaries which are better adapted to the data and also complexity of the data. Previous works [5,7] have also reported the effects of dictionary size in image classification. We investigate this in the particular case of classification.

We first describe sparse coding and dictionary learning in Section 2. The methodology is explained in Section 3, followed by results in Section 4 and conclusion in Section 5.

\section{SPARSE CODING AND DICTIONARY LEARNING}

Sparse coding is the process of finding a sparse coefficient vector $\mathbf{a} \in \mathbb{R}^{K}$ for representing a given signal $\mathbf{x} \in \mathbb{R}^{N}$ using a few atoms of an over-complete dictionary $\mathbf{D} \in \mathbb{R}^{N \times K}$. It is 
given by $\min _{\mathbf{a}}\|\mathbf{a}\|_{0}$ s.t. $\|\mathbf{x}-\mathbf{D a}\|_{2}^{2} \leq \varepsilon$, where $\|\cdot\|_{0}$ denotes the $l_{0}$ norm and $\varepsilon$ is the error in representation. Replacement of $l_{0}$ norm with the $l_{1}$ norm also results in sparse solution [8]. The sparse coding problem can then be given by

$$
\min _{\mathbf{a}}\|\mathbf{x}-\mathbf{D a}\|_{2}^{2}+\lambda\|\mathbf{a}\|_{1}
$$

where $\lambda$ balances the trade-off between the error and sparsity.

For a set of signals $\left\{\mathbf{x}_{i}\right\}_{i=1, ., m}$, we can find a dictionary $\mathbf{D}$ from the underlying data, such that each signal is represented by a sparse linear combination of its atoms. It is given by

$$
\min _{\mathbf{D},\left\{\mathbf{a}_{i}\right\}_{i=1, \ldots, m}} \sum_{i=1}^{m}\left\|\mathbf{x}_{i}-\mathbf{D} \mathbf{a}_{i}\right\|_{2}^{2}+\lambda\left\|\mathbf{a}_{i}\right\|_{1} .
$$

The optimization is an iterative two-step process: Sparse coding with fixed $\mathbf{D}$ and the dictionary update with fixed $\mathbf{a}$.

\section{PROPOSED APPROACH}

MR images from each patient are first processed for the removal of noise and non-brain tissues. The images are then registered and image patches of predefined size are extracted. These patches, after intensity normalization, are assigned to WM, GM, CSF or lesion class. For this purpose, we use manual lesion segmentation images and tissue segmentation obtained using SPM [9]. We then learn the dictionaries using training data for each class and perform patch based classification and voxel-wise classification for the given test subject. Each step is described in the subsections below.

\subsection{Pre-processing}

The noise introduced during MR acquisition is removed using non-local means and intensity inhomogenity correction [10, 11]. To ensure the spatial correspondence, the images are registered with respect to T1-w MPRAGE volume [12] and are processed further to extract the intra-cranial mask [13]. This limits the further analysis to the brain region.

\subsection{Patch Extraction and Labeling}

We divide the whole intracranial MR volume for each patient into 3-D patches, with a patch around every 2 voxels in each direction. The individual image patches of each MR modality are then flattened, concatenated together and are normalized. The patches are then labelled as either WM, GM, CSF or lesion class. If the number of lesion voxels in the corresponding image block of the manual segmentation image exceeds the pre-defined threshold $T_{L}$, we assign this patch to the lesion class. Otherwise, the patch is assigned to either WM, GM or CSF class, depending on the maximum number of voxels that belong to the corresponding class.

The labelled image patches are then divided into training and test data, and the experiments are performed by following Leave-One-Subject-Out-Cross-Validation (LOSOCV).

\subsection{Classification using Adaptive Dictionary Learning}

The class specific dictionaries are learned using training data of the corresponding class [14]. The subsections below describe different strategies adopted while learning these dictionaries and the scheme of patch based classification. In every method, we obtain the sparse codes for the test patches using Eq (1), knowing the dictionary $\mathbf{D}^{k}$ for the class $k$ [14].

\subsubsection{Two-Class: Same Dictionary Size (2-C SDS)}

Similar to [5], we use a single dictionary to represent the healthy class: WM, GM and CSF together. We learn the class specific dictionaries $\mathbf{D}^{k}$ of same size, for the healthy $(k=1)$ and lesion $(k=2)$ class. For a given test patch $\mathbf{y}_{\mathbf{i}}$, the classification is performed by calculating the sparse coefficients $\mathbf{a}_{i}^{k}$ for each class and the test patch is then assigned to class $c$ such that

$$
c=\underset{k}{\operatorname{argmin}}\left\|\mathbf{y}_{\mathbf{i}}-\mathbf{D}^{k} \mathbf{a}_{i}^{k}\right\|_{2}^{2} .
$$

\subsubsection{Two-Class: Different Dictionary Size (2-C DDS)}

In this method, we allow different dictionary sizes for the healthy and lesion classes, in order to take into consideration the data variability between classes and the number of training samples in each class. For accounting to more variability and larger training size, we allow larger dictionary size for the healthy class and obtain MS lesion classification.

\subsubsection{Four-Class: Same Dictionary Size (4-C SDS)}

The fact that every tissue, WM, GM and CSF, appears in different intensity pattern in each MR modality, using a single dictionary for representing tissues might not be as effective as learning separate dictionaries for each tissue. Each dictionary is representative of its own class and reconstruction of the test data using true class dictionary would give the minimum reconstruction error. We thus perform classification based on reconstruction error in a similar manner, as mentioned above.

\subsubsection{Four-Class: Different Dictionary Size (4-C DDS)}

Here, we experiment with different dictionary sizes for WM, GM, CSF and lesion classes, for similar reasons mentioned in Section 3.3.2.

\subsection{Voxel-wise Classification}

As already stated, we classify the patches centered around every 2 voxels in each direction. For voxel-wise classification, we assign each voxel to either of the classes by using majority voting. The voxel is assigned to a class using majority votes of all patches that contain the voxel.

In the context of lesion classification, we finally record the number of voxels that belong to True Positives (TP), False 
Negatives (FN) or False Positives (FP), and calculate sensitivity $(\mathrm{SEN})=\frac{T P}{T P+F N}$ and Positive Predictive Value (PPV) $=\frac{T P}{T P+F P}$.

\section{EXPERIMENTS AND RESULTS}

We evaluated the proposed approach on MR dataset of 14 MS patients acquired on a Verio 3T Siemens scanner. T1-w MPRAGE, T2-w, PD and FLAIR modalities were chosen for the analysis. The volume size for T1-w MPRAGE and FLAIR was $256 \times 256 \times 160$ and voxel size was $1 \times 1 \times 1 \mathrm{~mm}^{3}$. For T2$\mathrm{w}$ and $\mathrm{PD}$, the volume size was $256 \times 256 \times 44$ and voxel size was $1 \times 1 \times 3 \mathrm{~mm}^{3}$. Annotations of the lesions were carried out by an expert radiologist. One subject with strong MR artifacts was excluded from the analysis.

For labeling patches, we used threshold $T_{L}=6$, as mentioned in Section 3.2. For each subject, the number of lesion patches varied from $1 \mathrm{~K}$ to $30 \mathrm{~K}$, depending on the lesion load, whereas the average number of patches for WM, GM and CSF were $50 \mathrm{~K}, 90 \mathrm{~K}$ and $30 \mathrm{~K}$ respectively. Using LOSOCV, the patch size of $5 \times 5 \times 5$ and the sparsity parameter $\lambda=0.95$ were found to be optimal choices. We used these parameters for all experiments described next.

The voxel-wise classification results for the methods described above are shown in Table 1. Using same dictionary size of 5000 for healthy and lesion class, as denoted by method (a), results in high sensitivity but very low PPV. Considering more variability associated with the healthy class, we then use different dictionary sizes, 5000 for healthy and 1000 for lesion class. This drastically reduces FP, improving PPV, but also decreases sensitivity. We further enrich this model by learning tissue specific dictionaries for WM, GM, CSF. Using 4-class approach and the same dictionary size of 2000 for each tissue and lesion class gives better compromise between sensitivity and PPV. Finally, 4-class method with different dictionary sizes, 2000 each for WM, GM and CSF, and 1000 for lesion class, increases both the mean sensitivity and PPV values, as compared to the 2-class method with different dictionary sizes. The mean dice-scores for methods (b) and (d), as referred to in Table 1, are $43.46 \%$ and $48.20 \%$, respectively. Their comparison also shows a significant difference in PPV and dice-scores, with respective p-values of 0.0086 and 0.0298 . This confirms that learning class specific dictionaries for each tissue improves the classification.

The classification performance for various dictionary sizes in the 4-class methods is shown in Table 2. The results for method (c), in row X, show that the sensitivity and PPV increase until the dictionary sizes are increased to 2000. The dictionaries capture more details with the increase in their size, but sensitivity reduces thereafter, possibly because of the over-fitting in either class dictionary. In rows $\mathrm{Y}$ and $\mathrm{Z}$, we fix the size of either lesion or tissue dictionaries and vary the size of the other. It is observed that the best results, in terms of both sensitivity and PPV, are obtained for the dictionary

\begin{tabular}{|c|c|c|c|c|c|c|c|c|}
\hline Pat. & \multicolumn{2}{|c|}{ (a) } & \multicolumn{2}{c|}{ (b) } & \multicolumn{2}{c|}{ (c) } & \multicolumn{2}{c|}{$(\mathrm{d})$} \\
No. & \multicolumn{2}{|c|}{ 2-C SDS } & \multicolumn{2}{c|}{ 2-C DDS } & \multicolumn{2}{c|}{ 4-C SDS } & \multicolumn{2}{c|}{ 4-C DDS } \\
\hline & SEN & PPV & SEN & PPV & SEN & PPV & SEN & PPV \\
1 & 97 & 3 & 53 & 31 & 68 & 11 & 32 & 34 \\
2 & 98 & 2 & 66 & 41 & 78 & 9 & 60 & 38 \\
3 & 91 & 2 & 63 & 27 & 72 & 9 & 58 & 31 \\
4 & 98 & 17 & 57 & 68 & 89 & 50 & 69 & 83 \\
5 & 95 & 10 & 54 & 65 & 85 & 43 & 68 & 71 \\
6 & 89 & 29 & 38 & 55 & 80 & 48 & 56 & 64 \\
7 & 85 & 3 & 20 & 32 & 68 & 20 & 38 & 34 \\
8 & 98 & 3 & 69 & 21 & 89 & 10 & 72 & 24 \\
9 & 97 & 9 & 61 & 52 & 84 & 33 & 68 & 62 \\
10 & 98 & 12 & 66 & 41 & 91 & 29 & 73 & 47 \\
11 & 99 & 8 & 52 & 36 & 82 & 20 & 58 & 39 \\
12 & 100 & 3 & 77 & 31 & 91 & 11 & 72 & 30 \\
13 & 100 & 2 & 78 & 17 & 90 & 5 & 68 & 16 \\
\hline Avg & $\mathbf{9 5 . 8}$ & $\mathbf{7 . 9}$ & $\mathbf{5 8}$ & $\mathbf{3 9 . 8}$ & $\mathbf{8 2 . 1}$ & $\mathbf{2 2 . 9}$ & $\mathbf{6 0 . 9}$ & $\mathbf{4 4 . 1}$ \\
\hline
\end{tabular}

Table 1. Voxel-wise classification results using: (a) Two dictionaries with 5000 atoms for healthy and lesion classes (b) 5000 atoms for healthy and 1000 atoms for lesion class dictionary. (c) Four dictionaries with 2000 atoms for WM, GM, CSF and lesion class, (d) 2000 atoms for WM, GM and CSF, and 1000 atoms for lesion class dictionary.

size of 2000 for each tissue and 1000 for lesion class. It can also be observed that it is the relative dictionary size that drives the classification and is more important than just the absolute dictionary size for each class.

It is crucial to adapt the size of the dictionaries to better control the classification. For such purpose, we analyzed the data using Principal Component Analysis (PCA), which gives an estimate of the intrinsic dimensionality of the data. It was found that for each tissue, GM, WM and CSF, approximately twice as many eigenvalues are required for an arbitrary proportion of the cumulative data variance $(90 \%, 95 \%$ or $98 \%)$, as that required for the lesion data. Thus, as exhibited by method (d), it supports our adaption of the dictionary size for each tissue twice that for the lesion dictionary. For method (b), although the experimentally observed optimal dictionary size ratio of 5 was not found with PCA, the factor of 2 indicated by PCA still favors using higher dictionary size for the healthy class. One reasoning behind this might be the inability of PCA to analyze the non-linearity in the data. The intrinsic dimensionality estimation for this highly non-linear data could be further point of investigation.

In Figure 1, we show the results for patient 6, for all the methods discussed above. It can be seen that the methods (a) and (c), each using the dictionaries with the same size, suffer with many FP. The over-detections are reduced in methods (b) and (d), respectively, which use the adapted dictionary sizes. However, the 2-class method (b) has many FN. Including tissue specific information in the approach and using dictionaries of the adapted sizes results in significant improvement in the lesion classification with reduction in both FP and FN. This is shown in method (d), supporting our claim that the 


\begin{tabular}{|c|c|c|c|c|}
\hline & Tissues & Lesion & SEN & PPV \\
\hline & 500 & 500 & 81.1 & 17.2 \\
$\mathrm{X}$ & 1000 & 1000 & 81.8 & 19.7 \\
& 2000 & 2000 & 82.1 & 22.9 \\
& 5000 & 5000 & 80.6 & 25.5 \\
\hline \multirow{4}{*}{$\mathrm{Y}$} & 500 & 1000 & 94.9 & 5.2 \\
& 2000 & 1000 & 60.9 & 44.1 \\
& 5000 & 1000 & 30 & 67.5 \\
\hline \multirow{3}{*}{$\mathrm{Z}$} & 2000 & 500 & 32.7 & 65.1 \\
& 2000 & 1000 & 60.9 & 44.1 \\
& 2000 & 5000 & 95.3 & 6.5 \\
\hline
\end{tabular}

Table 2. Effect of dictionary sizes in classification. First two columns indicate the dictionary size for each tissue and lesion, whereas the last two columns indicate the sensitivity and PPV. Row X: Method (c) with various dictionary sizes, Rows Y and Z: Method (d) with fixed dictionary size for either lesion or tissues, while varying dictionary sizes for the other class.

method with the tissue specific dictionaries and adapted dictionary sizes is a better choice over the 2-class methods and those using the same dictionary size.

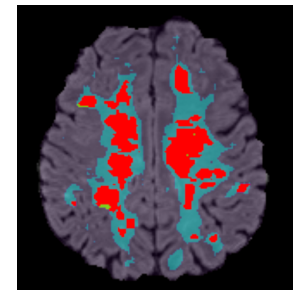

(a) 2C-SDS

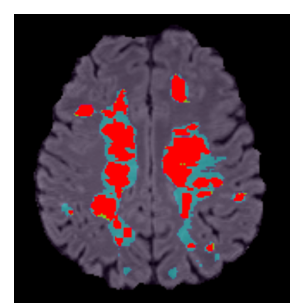

(c) 4C-SDS

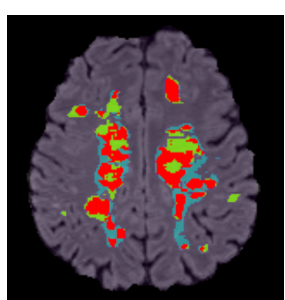

(b) 2C-DDS

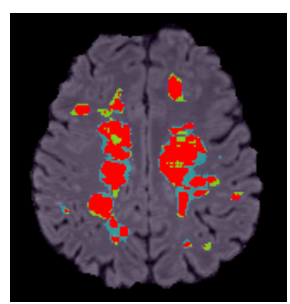

(d) 4C-DDS
Fig. 1. Comparison of MS lesion classification methods, as referred in Table 1. Classification image is superimposed on FLAIR MRI. TP are in red, FP are in cyan, FN are in green.

\section{CONCLUSION}

An approach for MS lesion classification based on dictionary learning and sparse representations is introduced in this work. Learning tissue specific dictionaries, instead of using a single dictionary for the non-lesion class, has shown clear improvement in the lesion classification. We have also demonstrated the effectiveness of adapting the dictionary sizes for better amplification of the differences among classes, hence improv- ing the classification. If performing PCA on input data can successfully adapt the dictionary size for the classification, it is not as much efficient when the classes represent more a mixture of different tissues. Knowing the limitation of PCA to handle only linear data, a future work will be to use the intrinsic dimension estimation techniques, which can better analyze complexity of the non-linear data.

\section{REFERENCES}

[1] M. Elad and M. Aharon, "Image denoising via learned dictionaries and sparse representation," in CVPR, June 2006, vol. 1, pp. 895-900.

[2] J. Wright, A. Yang, et al., "Robust face recognition via sparse representation," IEEE Trans. on PAMI, vol. 31, no. 2, pp. 210-227, Feb 2009.

[3] J. Mairal, J. Ponce, et al., "Supervised dictionary learning," in NIPS, pp. 1033-1040. 2009.

[4] N. Weiss et al., "Multiple sclerosis lesion segmentation using dictionary learning and sparse coding," in MICCAI 2013, vol. 8149, pp. 735-742. 2013.

[5] H. Deshpande et al., "Detection of multiple sclerosis lesions using sparse representations and dictionary learning," in MICCAI STMI Workshop, pp. 71-79. 2014.

[6] X. Lladó et al., "Segmentation of multiple sclerosis lesions in brain MRI: A review of automated approaches," Information Sci., vol. 186, no. 1, pp. 164 - 185, 2012.

[7] I. Ramirez and G. Sapiro, "An MDL framework for sparse coding and dictionary learning," IEEE Trans. on SP, vol. 60, no. 6, pp. 2913-2927, June 2012.

[8] S. Chen, D. Donoho, et al., "Atomic decomposition by basis pursuit," SISC, vol. 20, pp. 33-61, 1998.

[9] J. Ashburner and K. Friston, "Unified segmentation," NeuroImage, vol. 26, no. 3, pp. 839 - 851, 2005.

[10] N. Tustison, B. Avants, et al., "N4ITK: Improved N3 bias correction," IEEE TMI, vol. 29, no. 6, pp. 13101320, June 2010.

[11] P. Coupe et al., "Optimized blockwise nonlocal means denoising filter for 3-D magnetic resonance images," IEEE TMI, vol. 27, no. 4, pp. 425-441, April 2008.

[12] W. Wells III, P. Viola, et al., "Multi-modal volume registration by maximization of mutual information," Medical Image Analysis, vol. 1, no. 1, pp. 35 - 51, 1996.

[13] S. Smith, "Fast robust automated brain extraction," $\mathrm{Hu}$ man Brain Mapping, vol. 17, no. 3, pp. 143-155, 2002.

[14] J. Mairal, F. Bach, et al., "Online dictionary learning for sparse coding," in Proceedings of ICML, 2009. 